\title{
Using of Intraoperative Cell Salvage and Tranexamic Acid as Protective Factor for Postoperative Anemia Appearance in Patients with Total Hip or Knee Arthroplasty
}

\author{
Svetislav Matić $^{1, *}$, Mira Vuković ${ }^{2}$ and Aleksandar Vukićević ${ }^{3}$ \\ 1 Blood Bank Department, General Hospital Valjevo, 14000 Valjevo, Serbia \\ 2 Quality Assurance Department, General Hospital Valjevo, 14000 Valjevo, Serbia; vmira62@gmail.com \\ 3 Orthopedic Surgery Department, General Hospital Valjevo, 14000 Valjevo, Serbia; jocavrucina@gmail.com \\ * Correspondence: maticsv@gmail.com; Tel.: +38-16414129633
}

Citation: Matić, S.; Vuković, M.; Vukićević, A. Using of Intraoperative Cell Salvage and Tranexamic Acid as Protective Factor for Postoperative Anemia Appearance in Patients with Total Hip or Knee Arthroplasty. Surgeries 2021, 2, 308-319. https:// doi.org/10.3390/surgeries2030031

Academic Editor: Sibylle Kietaibl

Received: 5 July 2021

Accepted: 13 August 2021

Published: 16 August 2021

Publisher's Note: MDPI stays neutral with regard to jurisdictional claims in published maps and institutional affiliations.

Copyright: () 2021 by the authors. Licensee MDPI, Basel, Switzerland. This article is an open access article distributed under the terms and conditions of the Creative Commons Attribution (CC BY) license (https:// creativecommons.org/licenses/by/ $4.0 /)$.

\begin{abstract}
Background: The purpose of this study was to investigate intraoperative pharmacological and nonpharmacological methods and techniques in reducing blood loss in patients following total hip or knee arthroplasty. (2) Methods: A retrospective cross-sectional study was conducted in patients undergoing TKA or THA surgery, electively performed at the General Hospital Valjevo, Valjevo, Serbia, in 2014 when the principles of patient blood management (PBM) were not applied at all or in part, and in 2019 when PBM principles were applied as standard. (3) Results: This study includes 197 patients, of whom 83.8\% developed postoperative anemia (PA defined by haemoglobin $<12 \mathrm{~g} / \mathrm{dL}$ in both sexes). Using multivariate logistic regression and ROC curve analysis, it was shown that the use of tranexamic acid (TXA) with intraoperative cell salvage (ICS) in patients without preoperative anemia reduced the incidence of PA (odds ratio $=0.081)$. (4) Conclusions: Preoperative diagnosis and treatment of anemia are necessary in orthopedic patients since the use of TXA with ICS strongly reduces PA in patients without preoperative anemia.
\end{abstract}

Keywords: patient blood management; intraoperative cell salvage; tranexamic acid; postoperative anemia; total knee arthroplasty; total hip arthroplasty

\section{Introduction}

Anemia was defined by the World Health Organization (WHO) criteria as a hemoglobin $(\mathrm{Hb})$ concentration of less than $13 \mathrm{~g} / \mathrm{dL}$ in men and less than $12 \mathrm{~g} / \mathrm{dL}$ in women [1]. However, among the population older than 60 years, anemia, recently defined by levels of $\mathrm{Hb}<12 \mathrm{~g} / \mathrm{dL}$ in both sexes, is mostly of mild degree, from 10-12 g/dL [2]. Preoperative anemia is a common condition in surgical patients and is independently associated with increased perioperative and 30-day morbidity and mortality rate [3,4]. On the other hand, postoperative anemia (PA) is associated with poor postoperative outcomes, such as prolonged hospital stay, a higher incidence of postoperative renal failure and other organ dysfunction, and more frequent postoperative delirium due to reduced oxygen carrying capacity $[5,6]$.

Osteoarthrosis is a progressive, degenerative disease of joints, mostly affecting the hip and knee joints. The damage of cartilage can be caused by injury, aging or loading by heavy weight [7]. Major orthopedic operations such as total hip arthroplasty (THA) and total knee arthroplasty (TKA) can be associated with multiple complications [8]. In surgery for THA and TKA, major bleeding can occur and blood transfusion rates can be $45 \% \pm 25 \%$ and $44 \% \pm 15 \%$ respectively [9]. Beside benefits like better oxygenation and the correction of hematological status, transfusion of red blood cells (RBC) has many known complications. Errors in transfusion medicine, which most frequently involve misidentification of the patient, and may have life-threatening consequences [10]. There can also be issues with blood storage [11]. Patient blood management (PBM) is a strategy 
which applies several methods to reduce blood loss and transfusion, avoiding harm for the patients. The PBM system comprises a number of evidence-based interventions that can be applied before, during or after major surgery [12]. One way to save or recover red blood cells is the use of intraoperative cell salvage (ICS) devices. This is an important tool mostly used in cardiac, vascular and orthopedic surgery, where demands for blood products are high [13]. Moreover, by using this type of device, patients receive their own blood and risks from allogenic blood are reduced. An additional method for decreasing blood loss and lowering the risk of transfusion during surgical procedures is the use of antifibrinolytic drugs [14]. Another method for minimizing risk of bleeding during knee surgery is the use of a tourniquet. The tourniquet is a compressing device, used to control venous and arterial circulation to an extremity (lower extremity in TKA) for a period of time [15]. The introduction of PBM guidelines and their multidisciplinary strategies can improve patient outcomes [16]. According to European Society of Anesthesia guidelines, the use of cell saver devices, tranexamic acid (TXA), and techniques that minimize blood loss are highly recommended [17]. In accordance with the above, we considered it important in this study to assess the effects of demographic, anthropometric, hematological, and transfusion indicators, together with applied methods and techniques to prevent intraoperative and postoperative bleeding and postoperative anemia in patients undergoing knee or hip arthroplasty surgery.

\section{Materials and Methods}

This study was a retrospective, cross-sectional study in a cohort consisting of patients with elective, unilateral TKA or THA, hospitalized in Orthopedic Department of General Hospital Valjevo, Valjevo, Serbia. This study was approved by Ethics Committee of General Hospital Valjevo, Valjevo, Serbia, 01.06.2021. (OBV 01-4417/2021). Inclusion criteria were scheduled surgeries of elective, unilateral TKA or THA, performed by one of two chief surgeons with using: (1) operative approaches which minimize bleeding (tourniquet) alone, or (2) with cell saving techniques, or (3) with cell saving techniques in combination with TXA. Exclusion criteria were urgent surgeries, conditions that show a partial refractory response to TXA therapy in terms of blood loss and bleeding complications (diabetes mellitus) [18-20], thromboembolic events in near past, chronic cardiac disease, neurological diseases, or malignant disease.

The Data were collected from electronic patient database (Hospital Informatic System"Heliant"), and operative protocols for Department of Orthopedics and Blood bank. Patients operated during 2014 (with tourniquet and without the use of cell saving techniques or TXA, and for patients operated during 2019 (with tourniquet and with the use of cell saving techniques in combination with TXA). Cross-sectional analysis was performed between two groups of patients, divided on the basis of the presence/absence of PA. PA was defined as the occurrence of $\mathrm{Hb}<120 \mathrm{~g} / \mathrm{L}$ (in both sexes) in the postoperative period during hospitalization until the patient's discharge.

The blood count samples were collected preoperatively, marked as maximum $\mathrm{Hb}$ or hematocrit value. The minimal $\mathrm{Hb}$ or hematocrit value was the minimal level during hospital stay until the patient's discharge. Samples were analyzed by Abbott CELL-DYN Ruby analyzer [21]. ICS was performed with the "Xtra" device (Sorin, Italy). Blood aspirated from the operative field was mixed with unfractionated heparin 25,000 i.u. dissolved in $500 \mathrm{~mL}$ of saline solution $(0.9 \% \mathrm{NaCl})$, then filtered through a 40 -micron filter, and centrifuged up to $5600 \mathrm{rpm}$ according to manufacturer recommendations. Washing was performed with saline solution in a bowl with a volume of $125 \mathrm{~mL}$, with standard protocol of washing ('Pstd' $800 \mathrm{~mL}$ of saline solution for washing $300 \mathrm{~mL}$ of collected blood from operating field) according to device manufacturer [22]. Washed and salvaged RBC were reinfused through 170-micron filter for blood transfusion. All salvaged, washed RBC were reinfused in operating room at the end of surgical procedure in the operating theatre.

TXA was given in two doses. The first dose was applied as a bolus infusion $10 \mathrm{~min}$ before induction of general anesthesia in dose of $15 \mathrm{mg} / \mathrm{kg}$. The second dose was given 
on the first day after surgery in dose of $10 \mathrm{mg} / \mathrm{kg}$. All patients in the study underwent general endotracheal anesthesia (GEA). Induction in GEA was performed with propofol, and muscle relaxation was achieved using rocuronium bromide. The analgesia was with fentanyl citrate. The prescribed doses of drugs were adjusted according to the patient's body weight and according to the valid instructions for their use. Intraoperatively, continuous three-channel ECG monitoring was performed, with continuous monitoring of capillary blood saturation with oxygen as a non-invasive measurement of blood pressure at intervals not exceeding $5 \mathrm{~min}$. Hourly diuresis was also monitored. The duration of all surgical procedures was from 45 to $65 \mathrm{~min}$.

Tourniquet was induced $30 \mathrm{~min}$ before the end of surgical procedure with pressure of $300 \mathrm{~mm} \mathrm{Hg}$ and removed in moment of leaving operating room. All patients received routine thromboprophylaxis according to the recommendations from the European Society of Anesthesiology [23,24]. Dosage of thromboprophylactic drug Fraxiparine ${ }^{\circledR}$ (nadroparincalcium) was adjusted according to the patient's weight and the recommendations of Canadian manufacturer whose products are registered in Serbia. Fraxiparine ${ }^{\circledR}$ was given subcutaneously, $12 \mathrm{~h}$ before and $12 \mathrm{~h}$ after surgery, and then once daily to the third postoperative day: (1) for body weight $<50 \mathrm{~kg}-0.2 \mathrm{~mL}$ (Anti-Xa IU = $1900 \mathrm{IU}$ ); (2) for body weight from $50 \mathrm{~kg}$ to $69 \mathrm{~kg}-0.3 \mathrm{~mL}$ (Anti-Xa IU = $2850 \mathrm{IU}$ ) and (3) for body weight from $\geq 70 \mathrm{~kg}-0.4 \mathrm{~mL}$ (Anti-Xa IU = $3800 \mathrm{IU}$ ). From the fourth post-operative day onwards, for the next seven days, Fraxiparine was given once daily $(0.3 \mathrm{~mL}, 0.4 \mathrm{~mL}$ or $0.6 \mathrm{~mL})$, depending on the above body weight intervals ( $<50 \mathrm{~kg}$, from $50 \mathrm{~kg}$ to $69 \mathrm{~kg}, \geq 70 \mathrm{~kg}$ ). Demographic data of patients, height, weight, body mass index (BMI), hypertension, $\mathrm{Hb}$ maximum (preoperative), hematocrit maximum (preoperative), $\mathrm{Hb}$ minimum (lowest level in the postoperative period), hematocrit minimum (lowest level in the postoperative period), RBC salvaged volume, allogeneic RBC units, and Fresh Frozen Plasma (FFP) units (postoperative), and any complications, including postoperative thrombosis, re-bleeding, and wound infection or sepsis, until hospital discharge were documented. Estimated values for total blood volume and total RBC volume loss were also calculated for each patient [25] according to the following formulas:

$$
\text { PBV }=\mathrm{k} 1 \times \text { height }\left(\mathrm{m}^{3}\right)+\mathrm{k} 2 \times \text { weight }(\mathrm{kg})+\mathrm{k} 3
$$

where $\mathrm{k} 1=0.3669, \mathrm{k} 2=0.03219, \mathrm{k} 3=0.6041$ for men; and $\mathrm{k} 1=0.3561, \mathrm{k} 2=0.03308$, $\mathrm{k} 3=0.1833$ for women.

Total $\mathrm{RBC}$ volume loss $=\mathrm{PBV} \times($ Hematocrit maximum (preoperative) Hematocrit minimum (postoperative)

All patients were transfused according to recommendations of European Society of Anesthesia [26].

\subsection{Statistical Methods}

Continuous numerical data sets were described by the mean and standard deviation. The attributive or ordinal variables were described by the frequency of outcomes and percentages. Univariable analysis was performed using Pearson chi-square test or Fisher's exact test for categorical variables and Student $t$ test for continuous variables.

Binary logistic regression method with stepwise variable selection was used for multivariate analysis of postoperative anemia risk factors. All variables that had a $p<0.2$ on univariable analyses were considered for inclusion in the final model.

The evaluation of the validity of the logistic regression model implied an assessment of its goodness-of-fit measure and its accuracy. A goodness-of-fit model was made by estimating the Nagelkerke R Square. The accuracy of the logistic regression model was assessed using discrimination and adequacy. Discrimination measures were conducted to prove how adequately a model can distinguish patients with postoperative anemia from patients without postoperative anemia. The analysis of the adequacy of logistic models 
and the estimate of the retention of variables or their interactions was made using the Hosmer-Lemeshow method. Discrimination validity of scores of the newly constructed Post-Operative Anemia Risk Index (POARI) obtained by logistic regression model and other continuous variables in distinguish PA positive from PA negative patients was estimated by Receiver Operating Characteristic (ROC) procedure. "Cut point" value, sensitivity, specificity, positive predictive value, and negative predictive value were obtained by applying the maximum Youden index. The accepted level of significance was $p=0.05$. Statistical programs IBM SPSS Statistics for Windows, Version 22.0. Armonk, NY and MedCalc Software version 12.5.0.0 (MedCalc Software Ltd., Ostend, Belgium) were used for data processing.

\subsection{Development of POARI Score}

The number of appropriate points assigned to each variable equaled its regression coefficient divided by 0.5 , followed by rounding to the nearest whole number. The points for each risk factor were then summed to obtain the total number of points (score) for a patient.

\section{Results}

This study includes 197 orthopedic patients (46 male and 151 female), average age of 63.54 years \pm 8.58 years. Description of continuous variables is shown by (Table 1 ). From whole population of patients, $165(83.8 \%)$ developed PA. Compared to patents without $\mathrm{PA}$, patients with PA have lower values of weight, BMI, $\mathrm{Hb}$ maximum, $\mathrm{Hb}$ minimum, hematocrit maximum, hematocrit minimum, total blood volume, and RBC salvaged volume (Table 2). In addition, patients with PA have greater total RBC volume loss, more allogeneic RBC units, and more FFP unit transfusions compared to non-PA patients. With regard to other continuous variables, there was no difference between PA versus (vs.) non-PA patients.

Table 1. Descriptive statistics for continuous variables in the entire study sample of orthopedic patients.

\begin{tabular}{|c|c|c|c|}
\hline Variables & $\mathbf{n}$ & Mean & SD \\
\hline Age (years) & 197 & 63.54 & 8.58 \\
\hline Height (m) & 197 & 1.70 & 0.07 \\
\hline Weight (kg) & 197 & 77.25 & 12.10 \\
\hline Body mass index $\left(\mathrm{kg} / \mathrm{m}^{2}\right)$ & 197 & 26.52 & 3.32 \\
\hline Hemoglobin maximum (g/L) & 197 & 128.54 & 17.02 \\
\hline Hemoglobin minimum (g/L) & 197 & 101.65 & 18.27 \\
\hline Hematocrit maximum (\%) & 197 & 38.56 & 5.12 \\
\hline Hematocrit minimum (\%) & 197 & 30.47 & 5.51 \\
\hline Total blood volume (L) & 197 & 4.77 & 0.63 \\
\hline Total RBC volume loss (mL) & 197 & 386.41 & 241.16 \\
\hline RBC salvaged volume (mL) & 197 & 95.94 & 80.88 \\
\hline RBC (units) & 197 & 0.36 & 0.91 \\
\hline Fresh frozen plasma (units) & 197 & 0.08 & 0.34 \\
\hline Hospital days in the intensive care unit & 197 & 0.03 & 0.21 \\
\hline Hospital days in the semi-intensive care unit & 197 & 8.67 & 3.94 \\
\hline Hospital days in the ward & 197 & 5.96 & 5.13 \\
\hline Total hospital days & 197 & 14.66 & 5.93 \\
\hline Crystalloid solutions in the intensive care unit (bottles a $500 \mathrm{~mL}$ ) & 197 & 4.91 & 2.76 \\
\hline Crystalloid solution in the semi-intensive care unit (bottles a $500 \mathrm{~mL}$ ) & 197 & 8.02 & 4.92 \\
\hline Crystalloid solution in the ward (bottles a $500 \mathrm{~mL}$ ) & 197 & 0.64 & 2.41 \\
\hline Crystalloid solution throughout hospitalization (bottles a $500 \mathrm{~mL}$ ) & 197 & 13.57 & 6.93 \\
\hline
\end{tabular}

SD—standard deviation, n—number of patients, RBC—red blood cells, FFP—fresh frozen plasma, $\mathrm{n}=197$. 
Table 2. Assessment of differences between orthopedic patients without vs. with postoperative anemia according to continuous variables.

\begin{tabular}{|c|c|c|c|}
\hline \multirow{2}{*}{ Variables } & \multicolumn{2}{|c|}{$\begin{array}{l}\text { Postoperative Anemia } \\
\text { (Hemoglobin < } 120 \mathrm{~g} / \mathrm{L})\end{array}$} & \multirow{2}{*}{$\begin{array}{c}p \\
\text { (t Statistic) }\end{array}$} \\
\hline & $\begin{array}{l}\text { No }(\mathrm{n} 1=32) \\
\text { Mean } \pm \text { SD }\end{array}$ & $\begin{array}{l}\text { Yes }(\mathrm{n} 2=165) \\
\text { Mean } \pm \text { SD }\end{array}$ & \\
\hline Age (years) & $63.91 \pm 8.46$ & $63.47 \pm 8.62$ & $\begin{array}{c}0.794 \\
(0.261) \\
\end{array}$ \\
\hline Height (m) & $1.72 \pm 0.08$ & $1.70 \pm 0.07$ & $\begin{array}{c}0.148 \\
(1.454)\end{array}$ \\
\hline Weight (kg) & $84.00 \pm 12.81$ & $75.94 \pm 11.55$ & $\begin{array}{c}0.000 \\
(3.548)\end{array}$ \\
\hline Body mass index $\left(\mathrm{kg} / \mathrm{m}^{2}\right)$ & $28.30 \pm 3.31$ & $26.17 \pm 3.22$ & $\begin{array}{c}0.001 \\
(3.396)\end{array}$ \\
\hline Hemoglobin maximum (g/L) & $145.72 \pm 13.15$ & $125.21 \pm 15.65$ & $\begin{array}{c}0.000 \\
(7.813)\end{array}$ \\
\hline Hemoglobin minimum (g/L) & $127.56 \pm 7.25$ & $96.62 \pm 15.25$ & $\begin{array}{c}0.000 \\
(17.705\end{array}$ \\
\hline Hematocrit maximum (\%) & $43.69 \pm 3.92$ & $37.56 \pm 4.73$ & $\begin{array}{c}0.000 \\
(6.883) \\
\end{array}$ \\
\hline Hematocrit minimum (\%) & $38.27 \pm 2.17$ & $28.96 \pm 4.61$ & $\begin{array}{c}0.000 \\
(17.708)\end{array}$ \\
\hline Total blood volume (L) & $5.03 \pm 0.60$ & $4.72 \pm 0.62$ & $\begin{array}{c}0.012 \\
(2.540)\end{array}$ \\
\hline Total RBC volume loss (mL) & $272.40 \pm 161.23$ & $408.52 \pm 248.12$ & $\begin{array}{c}0.003 \\
(-) 3.953\end{array}$ \\
\hline RBC salvaged volume (mL) & $124.16 \pm 53.90$ & $90.47 \pm 84.16$ & $\begin{array}{c}0.005 \\
(2.913)\end{array}$ \\
\hline RBC (units) & $0.03 \pm 0.18$ & $0.42 \pm 0.99$ & $\begin{array}{c}0.000 \\
(-) 4.711\end{array}$ \\
\hline FFP (units) & $0.00 \pm 0.00$ & $0.09 \pm 0.38$ & $\begin{array}{c}0.002 \\
(-) 3.076\end{array}$ \\
\hline Total hospital days & $13.78 \pm 4.817$ & $14.83 \pm 6.11$ & $\begin{array}{c}0.361 \\
(-) 0.916\end{array}$ \\
\hline $\begin{array}{l}\text { Crystalloid solution during } \\
\text { hospitalization (bottles a } \\
500 \mathrm{~mL} \text { ) }\end{array}$ & $14.87 \pm 4.80$ & $13.31 \pm 7.26$ & $\begin{array}{c}0.245 \\
(1.166)\end{array}$ \\
\hline
\end{tabular}

SD—standard deviation, $n$ —number of patients, RBC—red blood cells, FFP—fresh frozen plasma.

With receiver operating characteristic (ROC) procedure we found three highly significant indicators that powerfully determine patients with PA versus non-PA. The criteria values of these indicators (variables) for the detection of patients with PA were: for weight $\leq 73 \mathrm{~kg}$, for total blood volume $\leq 4.544 \mathrm{~L}$, and for total RBC volume loss $>463.2 \mathrm{~mL}$ (Table 3). 
Table 3. Receiver operating characteristic (ROC) parameters for weight, total blood volume and total RBC volume loss in detection of patients with postoperative anemia after hip or knee arthroplasty.

\begin{tabular}{|c|c|c|c|c|c|c|c|}
\hline Variables & $\begin{array}{l}\text { AUROC } \\
(95 \% \text { CI })\end{array}$ & $p$ & Criterion & $\begin{array}{c}\mathrm{SN} \\
(95 \% \mathrm{CI})\end{array}$ & $\begin{array}{c}\mathrm{SP} \\
(95 \% \mathrm{CI})\end{array}$ & $\begin{array}{c}\text { PPV } \\
(95 \% \text { CI })\end{array}$ & $\begin{array}{c}\text { NPV } \\
(95 \% \text { CI })\end{array}$ \\
\hline $\begin{array}{l}\text { Weight } \\
\text { (kg) }\end{array}$ & $\begin{array}{c}0.677 \\
(0.607-0.742)\end{array}$ & 0.0003 & $\begin{array}{c}\leq 73 \\
(64-82)\end{array}$ & $\begin{array}{c}43.64 \\
(35.9-51.6)\end{array}$ & $\begin{array}{c}84.37 \\
(67.2-94.7)\end{array}$ & $\begin{array}{c}93.5 \\
(85.5-97.9)\end{array}$ & $\begin{array}{c}22.5 \\
(15.4-31.0)\end{array}$ \\
\hline $\begin{array}{l}\text { Total blood volume } \\
\text { (L) }\end{array}$ & $\begin{array}{c}0.649 \\
(0.578-0.715)\end{array}$ & 0.0016 & $\begin{array}{c}\leq 4.544 \\
(4.245-4.965)\end{array}$ & $\begin{array}{c}45.45 \\
(37.7-53.4)\end{array}$ & $\begin{array}{c}84.37 \\
(67.2-94.7)\end{array}$ & $\begin{array}{c}93.7 \\
(86.0-97.9)\end{array}$ & $\begin{array}{c}23.1 \\
(15.8-31.8)\end{array}$ \\
\hline $\begin{array}{l}\text { Total RBC volume } \\
\text { loss (mL) }\end{array}$ & $\begin{array}{c}0.653 \\
(0.582-0.719)\end{array}$ & 0.0021 & $\begin{array}{c}>463.2 \\
(069.5-484.5)\end{array}$ & $\begin{array}{c}37.58 \\
(30.2-45.4)\end{array}$ & $\begin{array}{c}93.75 \\
(79.2-99.2)\end{array}$ & $\begin{array}{c}96.9 \\
(89.1-99.6)\end{array}$ & $\begin{array}{c}22.6 \\
(15.8-30.6)\end{array}$ \\
\hline
\end{tabular}

AUROC—area under ROC curve, SN—sensitivity, SP—specificity, PPV—positive predictive value; NPV—negative predictive value, CI—confidence interval, RBC—red blood cells.

With univariate analyses, in comparison to non-PA patients, we have shown that patients with PA have less frequent ICS procedures plus TXA therapy ( $87.5 \%$ vs. $44.2 \%)$, frequently preoperative anemia $(100 \%$ vs. $66.7 \%)$, lower incidence of the absence of preoperative anemia in the subgroup with ICS + TXA $(87.5 \%$ vs $33.3 \%)$, less frequent increased body weight $(90.6 \%$ vs. $69.7 \%)$, less frequent $\mathrm{RBC}$ salvaged volume $>125 \mathrm{~mL}$ $(71.9 \%$ vs. $46.7 \%)$, less frequent patients with THA and RBC salvage $>125 \mathrm{~mL}(37.5 \%$ vs. $15.8 \%$ ) and less frequent presence of total blood volume $\leq 4.544 \mathrm{~L}(84.4 \%$ vs. $55.2 \%)$ (Table 4). On the other hand, in patients with PA compared to non-PA patients, the following characteristics are more common: total $\mathrm{RBC}$ volume loss $>463.2 \mathrm{~mL}$, weight $\leq 73 \mathrm{~kg}$, and more patients with TKA and with weight $\leq 73 \mathrm{~kg}$ (Table 4).

By applying multivariate regression analysis, we identified two independent risk factors and two protective interactions between predictors for PA and after which we made the allocation of appropriate points to form the POARI score (Table 5): (1) the interaction between group (ICS plus TXA) and preoperative anemia (no); (-)5 points; (2) total RBC volume loss > $463.2 \mathrm{~mL}$ (no); (-) 4 points; (3) total blood volume $\leq 4.544 \mathrm{~mL}$ (yes); +3 points and (4) the interaction between RBC salvaged volume $>125 \mathrm{~mL}$ (yes) and with TKA and weight $\leq 73 \mathrm{~kg}$ (yes); +5 points. The model above showed a moderate level of goodnessof-fit (Nagelkerke R Square $=0.455$ ) and very good adequacy (Hosmer-Lemeshow test Chi square $=3.098 ; p=0.876$ ) and discriminating characteristics (AUROC $=0.863$ with $95 \%$ confidence interval from 0.808 to $0.908, p=0.000, \mathrm{SN}=83.03 \%$ with $95 \%$ confidence interval from $91.1 \%$ to $88.4 \%, \mathrm{SP}=81.25 \%$ with confidence interval from $63.6 \%$ to $92.8 \%$, PPV $=95.8 \%$ with confidence interval from $91.1 \%$ to $98.4 \%$ and NPV $=48.1$ with $95 \%$ confidence interval from $34.3 \%$ to $62.2 \%$ ). POARI score $>-6$ with $95 \%$ confidence interval from -9 to -6 was the criteria for detection of very high PA risk (Table 5 and Figure 1). Average POARI score in the non-PA group of patients was $-7.50 \pm 2.54$, while in the group of patients with PA it was $-2.00 \pm 3.91$. 
Table 4. Univariate analysis of frequency distribution differences between orthopedic patients without vs. patients with postoperative anemia, according to nominal variables.

\begin{tabular}{|c|c|c|c|c|}
\hline \multirow{2}{*}{ Variables } & \multirow{2}{*}{ Categories } & \multicolumn{2}{|c|}{$\begin{array}{l}\text { Postoperative Anemia } \\
\text { (Hemoglobin }<120 \mathrm{~g} / \mathrm{L} \text { ) }\end{array}$} & \multirow{2}{*}{$\begin{array}{c}p \\
(\chi 2)\end{array}$} \\
\hline & & $\begin{array}{l}\text { No } \\
\text { f1 (\%) }\end{array}$ & $\begin{array}{l}\text { Yes } \\
\text { f2 (\%) }\end{array}$ & \\
\hline \multirow{3}{*}{ Group } & $\begin{array}{l}\text { without ICS or } \\
\text { TXA }\end{array}$ & $4(12.5 \%)$ & $64(38.8 \%)$ & \multirow{3}{*}{$\begin{array}{c}0.000 \\
(20.578)\end{array}$} \\
\hline & only ICS & $0(0.0 \%)$ & $28(17.0 \%)$ & \\
\hline & ICS and TXA & $28(87.5 \%)$ & $73(44.2 \%)$ & \\
\hline \multirow{2}{*}{ Gender } & male & $11(34.4 \%)$ & $35(21.2 \%)$ & \multirow{2}{*}{$\begin{array}{c}0.107 \\
(2.595)\end{array}$} \\
\hline & female & $21(65.6 \%)$ & $130(78.8 \%)$ & \\
\hline \multirow{2}{*}{ Orthopedic surgery } & THA & $12(37.5 \%)$ & $72(43.6 \%)$ & \multirow{2}{*}{$\begin{array}{c}0.521 \\
(0.413)\end{array}$} \\
\hline & TKA & $20(62.5 \%)$ & $93(56.4 \%)$ & \\
\hline \multirow{2}{*}{$\begin{array}{l}\text { Preoperative anemia } \\
\text { (hemoglobin }<120 \mathrm{~g} / \mathrm{L} \text { ) }\end{array}$} & No & $32(100.0 \%)$ & $110(66.7 \%)$ & \multirow{2}{*}{0.000} \\
\hline & Yes & $0(0.0 \%)$ & $55(33.3 \%)$ & \\
\hline \multirow{2}{*}{$\begin{array}{c}\text { Absence of preoperative anemia } \\
\text { (hemoglobin }<120 \mathrm{~g} / \mathrm{L} \text { ) and without } \\
\text { ICS or TXA }\end{array}$} & No & $28(87.5 \%)$ & $125(75.8 \%)$ & \multirow{2}{*}{0.170} \\
\hline & Yes & $4(12.5 \%)$ & $55(33.3 \%)$ & \\
\hline \multirow{2}{*}{$\begin{array}{c}\text { Absence of preoperative anemia } \\
\text { (hemoglobin }<120 \mathrm{~g} / \mathrm{L} \text { ) and only ICS }\end{array}$} & No & $32(100.0 \%)$ & $150(90.9 \%)$ & \multirow{2}{*}{0.137} \\
\hline & Yes & $0(0.0 \%)$ & $15(9.1 \%)$ & \\
\hline \multirow{2}{*}{$\begin{array}{c}\text { Absence of preoperative anemia } \\
\text { (hemoglobin }<120 \mathrm{~g} / \mathrm{L} \text { ) and ICS with } \\
\text { TXA }\end{array}$} & No & $4(12.5 \%)$ & $110(66.7 \%)$ & \multirow{2}{*}{0.000} \\
\hline & Yes & $28(87.5 \%)$ & $55(33.3 \%)$ & \\
\hline \multirow{2}{*}{ Older (greater or equal 60 years) } & No & $7(21.9 \%)$ & $47(28.5 \%)$ & \multirow{2}{*}{0.521} \\
\hline & Yes & $25(78.1 \%)$ & $118(71.5 \%)$ & \\
\hline \multirow{2}{*}{$\begin{array}{l}\text { Increased body weight (body mass } \\
\text { index } \geq 25 \mathrm{~kg} / \mathrm{m}^{2} \text { ) }\end{array}$} & No & $3(9.4 \%)$ & $50(30.3 \%)$ & \multirow{2}{*}{0.016} \\
\hline & Yes & $29(90.6 \%)$ & $115(69.7 \%)$ & \\
\hline \multirow{2}{*}{ Hypertension } & No & $20(62.5 \%)$ & $100(60.6 \%)$ & \multirow{2}{*}{$\begin{array}{c}0.841 \\
(0.040)\end{array}$} \\
\hline & Yes & $12(37.5 \%)$ & $65(39.4)$ & \\
\hline \multirow{2}{*}{ RBC salvaged volume $>125 \mathrm{~mL}$} & No & $9(28.1 \%)$ & $88(53.3 \%)$ & \multirow{2}{*}{0.012} \\
\hline & Yes & $23(71.9 \%)$ & $77(46.7 \%)$ & \\
\hline \multirow{2}{*}{$\begin{array}{c}\text { Patients with TKA and RBC salvaged } \\
\text { volume }>125 \mathrm{~mL}\end{array}$} & No & $21(65.6 \%)$ & $114(69.1 \%)$ & 0.149 \\
\hline & Yes & $11(34.4 \%)$ & $51(30.9 \%)$ & $(0.699)$ \\
\hline Patients with THA and RBC salvaged & No & $20(62.5 \%)$ & $139(84.2 \%)$ & 0007 \\
\hline volume $>125 \mathrm{~mL}$ & Yes & $12(37.5 \%)$ & $26(15.8 \%)$ & 0.007 \\
\hline & No & $30(93.8 \%)$ & $103(62.4 \%)$ & 0 \\
\hline lotal KBC volume loss > 463.2 mL & Yes & $2(6.2 \%)$ & $62(37.6 \%)$ & 0.000 \\
\hline 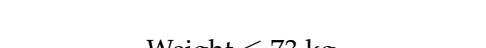 & No & $27(84.4 \%)$ & $93(56.4 \%)$ & קחבח \\
\hline Weight $\leq 73 \mathrm{~kg}$ & Yes & $5(15.6 \%)$ & $72(43.6 \%)$ & 0.003 \\
\hline Patients with TKA and with & No & $28(87.5 \%)$ & $114(69.1 \%)$ & 0000 \\
\hline weight $\leq 73 \mathrm{~kg}$ & Yes & $4(12.5 \%)$ & $51(30.9 \%)$ & 0.035 \\
\hline Patients with THA and with & No & $31(96.9 \%)$ & $144(87.3 \%)$ & 0126 \\
\hline weight $\leq 73 \mathrm{~kg}$ & Yes & $1(3.1 \%)$ & $21(12.7 \%)$ & 0.136 \\
\hline 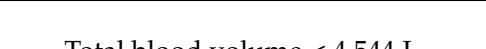 & No & $27(84.4 \%)$ & $91(55.2 \%)$ & 0002 \\
\hline Iotal Diood vorume $<4.344 \mathrm{~L}$ & Yes & $5(15.6 \%)$ & $74(44.8 \%)$ & 0.000 \\
\hline
\end{tabular}

f-frequency, RBC—red blood cells, ICS—intraoperative cell salvage, TKA—total knee arthroplasty, THA—total hip arthroplasty, TXA—-tranexamic acid. 
Table 5. Parameters for PA predictors in multivariate logistic regression model with appropriate points for Post-Operative Index score construction in patients with hip or knee arthroplasty.

\begin{tabular}{|c|c|c|c|c|c|c|c|c|}
\hline \multirow[t]{2}{*}{ Parameters for PA Predictors } & \multirow[t]{2}{*}{ B } & \multirow[t]{2}{*}{ SE } & \multirow[t]{2}{*}{ Wald } & \multirow[t]{2}{*}{$p$} & \multirow{2}{*}{$\begin{array}{l}\text { Odds } \\
\text { Ratio }\end{array}$} & \multicolumn{2}{|c|}{$\begin{array}{l}\text { 95\% CI for Odds } \\
\text { Ratio }\end{array}$} & \multirow[t]{2}{*}{ Points } \\
\hline & & & & & & Lower & Upper & \\
\hline Group by preoperative anemia & & & 18.234 & 0.000 & & & & \\
\hline $\begin{array}{l}\text { Group (without ICS and TXA) by } \\
\text { preoperative anemia } \\
\text { (no)-Reference }\end{array}$ & - & - & - & - & - & - & - & 0 \\
\hline $\begin{array}{c}\text { Group (only ICS) by preoperative } \\
\text { anemia (no) }\end{array}$ & 17.643 & 9543.105 & 0.000 & 0.999 & 45957783.656 & 0.000 & - & 0 \\
\hline $\begin{array}{l}\text { Group (ICS plus TXA) by } \\
\text { preoperative anemia (no) }\end{array}$ & -2.517 & 0.589 & 18.234 & 0.000 & 0.081 & 0.025 & 0.256 & -5 \\
\hline $\begin{array}{l}\text { Total RBC volume loss }>463.2 \mathrm{~mL} \\
\text { (yes)-Reference }\end{array}$ & - & - & - & - & - & - & - & 0 \\
\hline $\begin{array}{l}\text { Total RBC volume loss }>463.2 \mathrm{~mL} \\
\text { (no) }\end{array}$ & -2.165 & 0.795 & 7.418 & 0.006 & 0.115 & 0.024 & 0.545 & -4 \\
\hline $\begin{array}{l}\text { Total blood volume }<4.544 \mathrm{~mL} \\
\text { (no)-Reference }\end{array}$ & - & - & - & - & - & - & - & 0 \\
\hline $\begin{array}{c}\text { Total blood volume }<4.544 \mathrm{~mL} \\
\text { (yes) }\end{array}$ & 1.385 & 0.575 & 5.792 & 0.016 & 3.993 & 1.293 & 12.333 & 3 \\
\hline $\begin{array}{l}\text { RBC salvaged volume }>125 \mathrm{~mL} \\
\text { (no) by patients TKA with weight } \\
\leq 73 \mathrm{~kg} \text { (no)-Reference }\end{array}$ & - & - & - & - & - & - & - & 0 \\
\hline $\begin{array}{l}\text { RBC salvaged volume }>125 \mathrm{~mL} \\
\text { (yes) by patients with TKA and } \\
\text { with weight } \leq 73 \mathrm{~kg} \text { (yes) }\end{array}$ & 2.289 & 1.087 & 4.437 & 0.035 & 9.864 & 1.173 & 82.973 & 5 \\
\hline Constant & 4.352 & 0.886 & 24.131 & 0.000 & 77.665 & & & \\
\hline
\end{tabular}

SE—standard error, CI—confidence interval, B-regression coefficient, TKA—total knee prosthesis, ICS—intraoperative cell salvage, TXA—tranexamic acid, RBC-red blood cells.

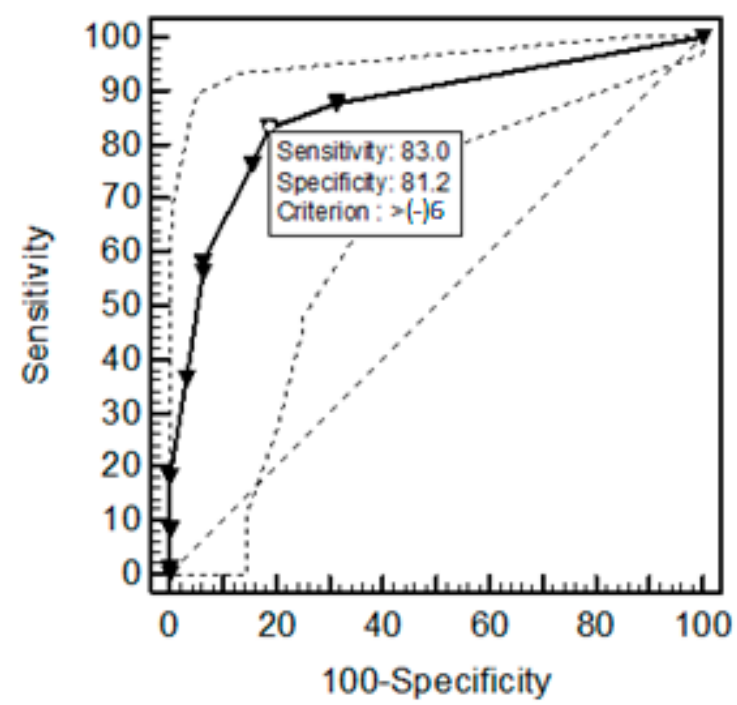

Figure 1. ROC curve for Post-Operative Anemia Risk Index score in orthopedic patients with hip or knee arthroplasty. 


\section{Discussion}

In our study, the combined effects of demographic, anthropometric, and hematological predictors of postoperative anemia were assessed, together with the use of various pharmacological and/or non-pharmacological procedures for the control of intra- and postoperative bleeding in patients with THA or TKA.

With multivariate logistic regression analysis, we have shown that in orthopedic patients undergoing THA or TKA surgery, without preoperative anemia, the use of TXA in combination with the ICS procedure achieves a protective effect on PA development (odds ratio $=0.081)($ Table 5$)$. Hence, in order to eliminate preoperative anemia, the early preoperative differential diagnosis of anemia and its appropriate therapy can significantly reduce the incidence of PA in patients undergoing THA or TKA surgery, in whom ICS and TXA would be administered intraoperatively. The European Association of Anesthesiology recommends, 4- 8 weeks before the procedure, oral or intravenous iron supplementation and/or erythropoietin therapy to surgery patients with preoperative anemia in whom nutritional deficiencies are absent or have been corrected along with a restrictive transfusion policy [26]. Note that The European Association of Anesthesiology recommends erythropoietin therapy only in cases of absence of the target therapeutic effect (achieving $\mathrm{Hb} \geq 13 \mathrm{~g} / \mathrm{dL}$ ) with iron supplementation.

Independent of the protective factors for PA discussed above, in patients with THA or TKA, only in the absence of total RBC volume loss $>463.2 \mathrm{~mL}$, the risk for PA is significantly lower (odds ratio $=0.115$ ). As total $\mathrm{RBC}$ volume loss is (Formula 2 ) dependent on the difference between maximum and minimum values of hematocrit in blood count, patients without total RBC volume loss $>463.2 \mathrm{~mL}$ have smaller amount of blood loss intra- and postoperatively comparing to preoperative values of hematocrit. According to this data, we suggest that in goal to evaluate of total protective factors in patients with THA or TKA, routine monitoring of total $\mathrm{RBC}$ volume loss is mandatory during time of hospitalization. Brecher et al. [27] have shown that use of mathematical modeling can rapidly estimate a patient's blood loss and allows more judicial and informed decision on what (if any) blood conservation techniques are to be employed in a specific patient. POARI $>-6$ score is criteria for the risk of developing PA. In the absence of risk factors as specified in the multivariate logistic model (Table 5), orthopedic patients with presence of both protective factors (absence of preoperative anemia and using of ICS with TXA and absence of total volume loss $>463.2 \mathrm{~mL}$ ), will not have PA with a probability of $81.2 \%$ (POARI score $=-9$ ). Muñoz et al. [28], in their review and recommendations, show that orthopedic patients at risk of developing severe postoperative anemia should be identified on the basis of RBC mass (reflected by $\mathrm{Hb}$ concentration on the day of preoperative assessment), the lowest $\mathrm{Hb}$ concentration that the patient can tolerate (transfusion trigger), and the expected blood loss. However, even in cases where both protective factors are present (Table 5), patients with THA or TKA will develop PA with probability of $83 \%$ in case of the total blood volume $<4.544 \mathrm{~mL}$ (POARI score $=-6$ ). These patients are candidates for early PA treatment with intravenous iron therapy as early as possible during the period of hospitalization and home recovery. Patients of short height and low body weight (Formula (1)), with a reduced level of circulating volume, and their potential intraoperative blood loss, in proportion to their circulating volume, can be potentially higher and can result in PA. Kotzé et al. [29] described that the early use of intravenous iron can improve outcomes of orthopedic patients developing PA, allogeneic blood transfusion, increased hospital stay, or re-admission. Desai et al. [30] emphasize that, before a surgical procedure, "females should be optimized to the same level of $\mathrm{Hb}$ as males to $13 \mathrm{~g} / \mathrm{dL}$ since both sexes lose comparable amounts of blood in similar surgical procedures, but females have a relatively lower circulating volume which means they lose proportionally more".

Patients with only TKA will have four times higher risk for PA (Table 5) if their weight is $\leq 73 \mathrm{~kg}$ and blood salvaged $>125 \mathrm{~mL}$. Vieira et al. [31] have shown that ICS device has a high recovery rate of RBC and high percentage of eliminating residual heparin, plasma proteins, albumins, and platelets. In procedures with ICS, the salvaged component is RBC only, 
so patients with $\mathrm{RBC}$ salvaged volume $>125 \mathrm{~mL}$ can have significant intraoperative loss of platelets and coagulation factors. In this patient group, risk of prolonged postoperative bleeding is high, specifically in ordinating fixed doses of Low Molecular Weight Heparin (LMWH) for preventing thromboembolic events without concern of their weight. Thus, to avoid more postoperative bleeding at patients with TKA and weight $\leq 73 \mathrm{~kg}$ combined with a volume of salvaged $\mathrm{RBC}$ volume greater than $125 \mathrm{~mL}$, we recommend $\mathrm{LMWH}$ therapy in preventing doses for thromboembolic events in accordance with their weight. In their meta-analysis, Smith et al. [32] described greater intra-operative blood loss in nontourniquet compared to tourniquet assisted surgery, although they found no difference in total blood loss or transfusion rate. However, in our study, we showed that total RBC volume loss and intraoperative blood loss were independent predictors of PA (Table 5). In our study, because the above risk factor for postoperative anemia was significant only in patients with TKA, in order to more adequately control and reduce intraoperative blood loss to $125 \mathrm{~mL}$, we consider the use of tourniquet during the first half of the operation (not until its end) and/or additional intraoperative topical application of TXA as potentially useful preventive procedures.

Many studies have shown that patients with PA have prolonged hospital stay [6]. With univariate analysis we found no difference in days of hospital stay between patients with PA vs. non-PA patients (Table 2). According to our findings, the use of RBC and FFP was higher in the group of patients with PA vs. non-PA, and we have observed that, in both groups, the policy regarding the use of RBC and FFP was restrictive in accordance with European Society of Anesthesiology recommendations [25,33].

Limitations of this study are a single center and retrospective design, specifically a relatively small sample size that is particularly relevant to the group of patients without PA. In connection with the above, we defined anemia, in both sexes, as $\mathrm{Hb}<12 \mathrm{~g} / \mathrm{dL}$, since we predominantly considered old patients $(72.6 \%)$, which is inconsistent with the WHO definition of anemia [1]. Moreover, in the study population, we did not record the chronic use of non-steroidal anti-inflammatory drugs, for which The European Association of Anesthesiology has made special recommendations due to the connection of their application with major or prolonged intraoperative and/or postoperative bleeding in orthopedic surgery [26]. In accordance with the mentioned recommendations, in the population of patients with preoperative anemia, we did not record the type of anemia according to its cause.

\section{Conclusions}

Using patient blood management principles and guidelines, intraoperative cell salvage and the use of tranexamic acid represent a powerful protective factor to prevent postoperative anemia, exclusively in the patient population without preoperative anemia. Routine monitoring of red blood cell total volume loss is mandatory during hospitalization, since the absence of total red blood cell volume loss $>463.2 \mathrm{~mL}$ reduces the chance of developing postoperative anemia. The total blood volume $<4544 \mathrm{~mL}$ represents a serious risk for the development of postoperative anemia, and therefore orthopedic patients with the mentioned finding should optimize the level of hemoglobin, in both sexes, in order to be $\geq 13 \mathrm{~g} / \mathrm{dl}$. Further research is needed to investigate more aspects of risk factors and the prevention of postoperative anemia in orthopedic patients.

Author Contributions: Conceptualization, S.M.; methodology, S.M.; software, M.V.; validation, S.M., M.V. and A.V.; formal analysis, S.M. and M.V.; investigation, S.M. and A.V.; resources, A.V.; data curation, S.M.; writing - original draft preparation, S.M. and M.V. All authors have read and agreed to the published version of the manuscript.

Funding: This research received no external funding.

Institutional Review Board Statement: The study was conducted according to the guidelines of the Declaration of Helsinki, and approved by the Ethics Committee of General Hospital Valjevo, Valjevo, Serbia (OBV 01-4417/2021), 1 June 2021. 
Informed Consent Statement: Not applicable.

Data Availability Statement: The Data were collected from electronic patient database (Hospital Informatic System-“Heliant”), Regional Hospital Valjevo, Serbia.

Acknowledgments: Authors would like to thank Nick Schofield, for editing manuscript in English as mother speaking editor.

Conflicts of Interest: The authors declare no conflict of interest.

$\begin{array}{ll}\text { Abbreviations } \\ \text { WHO } & \text { World Health Organization } \\ \text { Hb } & \text { Hemoglobin } \\ \text { THA } & \text { total hip arthroplasty } \\ \text { TKA } & \text { total knee arthroplasty } \\ \text { PBM } & \text { Patient Blood Management } \\ \text { ICS } & \text { Intraoperative cell salvage } \\ \text { TXA } & \text { Tranexamic acid } \\ \text { RBC } & \text { Red blood cells } \\ \text { POARI } & \text { Post-Operative Anemia Risk Index } \\ \text { ROC } & \text { Receiver Operating Characteristic } \\ \text { PA } & \text { postoperative anemia } \\ \text { BMI } & \text { Body Mass Index } \\ \text { FFP } & \text { Fresh Frozen Plasma } \\ \text { vs. } & \text { versus } \\ \text { LMWH } & \text { Low Molecular Weight Heparin }\end{array}$

\section{References}

1. WHO Scientific Group. Nutritional Anemias. Report of a WHO Group of Experts. World Health Organ. Tech. Rep. Ser. 1972, 503, $1-29$.

2. Cappellini, M.D.; Motta, I. Anemia in Clinical Practice-Definition and Classification: Does Hemoglobin Change With Aging? Semin. Hematol. 2015, 52, 261-269. [CrossRef] [PubMed]

3. Musallam, K.M.; Tamim, H.M.; Richards, T.; Spahn, D.R.; Rosendaal, F.R.; Habbal, A.; Khreiss, M.; Dahdaleh, F.S.; Khavandi, K.; Sfeir, P.M.; et al. Preoperative anaemia and postoperative outcomes in non-cardiac surgery: A retrospective cohort study. Lancet 2011, 378, 1396-1407. [CrossRef]

4. Fowler, A.J.; Ahmad, T.; Phull, M.K.; Allard, S.; Gillies, M.A.; Pearse, R.M. Meta-analysis of the association between preoperative anaemia and mortality after surgery. Br. J. Surg. 2015, 102, 1314-1324. [CrossRef] [PubMed]

5. Hobson, C.; Ozrazgat-Baslanti, T.; Kuxhausen, A.; Thottakkara, P.; Efron, P.A.; Moore, F.A.; Moldawer, L.L.; Segal, M.S.; Bihorac, A. Cost and Mortality Associated With Postoperative Acute Kidney Injury. Ann. Surg. 2015, 261, 1207-1214. [CrossRef]

6. Kunz, J.V.; Spies, C.D.; Bichmann, A.; Sieg, M.; Mueller, A. Postoperative anaemia might be a risk factor for postoperative delirium and prolonged hospital stay: A secondary analysis of a prospective cohort study. PLoS ONE 2020, 15, e0229325. [CrossRef]

7. Felson, D.T. An update on the pathogenesis and epidemiology of osteoarthritis. Radiol. Clin. N. Am. 2004, 42, 1-9.

8. Fuji, T.; Akagi, M.; Abe, Y.; Oda, E.; Matsubayashi, D.; Ota, K.; Kobayashi, M.; Matsushita, Y.; Kaburagi, J.; Ibusuki, K.; et al. Incidence of venous thromboembolism and bleeding events in patients with lower extremity orthopedic surgery: A retrospective analysis of a Japanese healthcare database. J. Orthop. Surg. Res. 2017, 12, 55. [CrossRef]

9. Spahn, R.D. Anemia and Patient Blood Management in Hip and Knee Surgery. A Systematic Review of the Literature. Anesthesiology 2010, 113, 482-495. [CrossRef]

10. Massimo, F. Errors in transfusion: Causes and measures to avoid them. Clin. Chem. Lab. Med. 2010, 48, $1075-1077$.

11. Roberts, N.; James, S.; Delaney, M.; Fitzmaurice, C. The global need and availability of blood products: A modelling study. Lancet Haematol. 2019, 6, e606-e615. [CrossRef]

12. Kietaibl, S. Facilitating the Implementation of Perioperative Patient Blood Management: Education, Infrastructure, Process Descriptions, Quality Indicators and Patient Information. Austin J. Anesth. Analg. 2019, 7, 1079.

13. Sikorski, R.A.; Rizkalla, N.A.; Yang, W.W.; Frank, S.M. Autologous blood salvage in the era of patient blood management. Vox Sang. 2017, 112, 499-510. [CrossRef] [PubMed]

14. Bisbe, E.; Moltó, L. Pillar 2: Minimising bleeding and blood loss. Best Pract. Res. Clin. Anaesthesiol. 2013, 27, 99-110. [CrossRef]

15. Themistoklis, T.; Theodosia, V.; Konstantinos, K.; Georgios, D.I. Perioperative blood management strategies for patients undergoing total knee replacement: Where do we stand now? World J. Orthop. 2017, 8, 441-454. [CrossRef] 
16. Muñoz, M.; Gómez-Ramírez, S.; Kozek-Langeneker, S.; Shander, A.; Richards, T.; Pavía, J.; Kehlet, H.; Acheson, A.G.; Evans, C.; Raobaikady, R.; et al. 'Fit to fly': Overcoming barriers to preoperative haemoglobin optimization in surgical patients. Br. J. Anaesth. 2015, 115, 15-24. [CrossRef] [PubMed]

17. Kozek-Langenecker, S.A.; Imberger, G.; Rahe-Meyer, N.; Afshari, A. Reply to: ESA guidelines on the management of severe perioperative bleeding. Eur. J. Anaesthesiol. 2014, 31, 241-243. [CrossRef] [PubMed]

18. Myles, P.S.; Smith, J.A.; Forbes, A.; Silbert, B.; Jayarajah, M.; Painter, T.; Cooper, D.J.; Marasco, S.; McNeil, J.; Bussières, J.S.; et al. Tranexamic Acid in Patients Undergoing Coronary-Artery Surgery. N. Engl. J. Med. 2017, 376, 136-148. [CrossRef]

19. Alzahrani, S.H.; Ajjan, R.A. Coagulation and fibrinolysis in diabetes. Diabetes Vasc. Dis. Res. 2010, 7, 260-273. [CrossRef]

20. Ajjan, R.A.; Gamlen, T.; Standeven, K.F.; Mughal, S.; Hess, K.; Smith, K.A.; Dunn, E.J.; Anwar, M.M.; Rabbani, N.; Thornalley, P.J.; et al. Diabetes is associated with posttranslational modifications in plasminogen resulting in reduced plasmin generation and enzyme-specific activity. Blood 2013, 122, 134-142. [CrossRef] [PubMed]

21. Leers, M.P.; Goertz, H.; Feller, A.; Hoffmann, J.J. Performance evaluation of the Abbott CELL-DYN Ruby and the Sysmex XT-2000i haematology analysers. Int. J. Lab. Hematol. 2011, 33, 19-29. [CrossRef]

22. Overdevest, E.P.; Lanen, P.W.; Feron, J.C.; van Hees, J.W.; Tan, M.E. Clinical evaluation of the Sorin Xtra(R) Autotransfusion System. Perfusion 2012, 27, 278-283. [CrossRef]

23. Afshari, A.; Ageno, W.; Ahmed, A.; Duranteau, J.; Faraoni, D.; Kozek-Langenecker, S.; Llau, J.; Nizard, J.; Solca, M.; Stensballe, J.; et al. ESA VTE Guidelines Task Force. European Guidelines on perioperative venous thromboembolism prophylaxis: Executive summary. Eur. J. Anaesthesiol. 2018, 35, 77-83. [CrossRef] [PubMed]

24. Kozek-Langenecker, S.; Fenger-Eriksen, C.; Thienpont, E.; Barauskas, G. European guidelines on perioperative venous thromboembolism prophylaxis: Surgery in the elderly. Eur. J. Anaesthesiol. 2018, 35, 116-122. [CrossRef] [PubMed]

25. Sehat, K.R.; Evans, R.; Newman, J.H. How much blood is really lost in total knee arthroplasty? Correct blood loss management should take hidden loss into account. Knee 2000, 7, 151-155. [CrossRef]

26. Kozek-Langenecker, S.A.; Ahmed, A.B.; Afshari, A.; Albaladejo, P.; Aldecoa, C.; Barauskas, G.; De Robertis, E.; Faraoni, D.; Filipescu, D.C.; Fries, D.; et al. Management of severe perioperative bleeding: Guidelines from the European Society of Anaesthesiology: First update 2016. Eur. J. Anaesthesiol. 2017, 34, 332-395. [CrossRef] [PubMed]

27. Brecher, M.E.; Monk, T.; Goodnough, L.T. A standardized method for calculating blood loss. Transfusion 1997, 37, 1070-1074. [CrossRef] [PubMed]

28. Muñoz, M.; García-Erce, J.A.; Villar, I.; Thomas, D. Blood conservation strategies in major orthopaedic surgery: Efficacy, safety and European regulations. Vox Sang. 2009, 96, 1-13. [CrossRef] [PubMed]

29. Kotzé, A.; Carter, L.A.; Scally, A.J. Effect of a patient blood management programme on preoperative anaemia, transfusion rate, and outcome after primary hip or knee arthroplasty: A quality improvement cycle. Br. J. Anaesth. 2012, 108, 943-952. [CrossRef] [PubMed]

30. Desai, N.; Schofield, N.; Richards, T. Perioperative Patient Blood Management to Improve Outcomes. Anesth. Analg. 2018, 127, 1211-1220. [CrossRef]

31. Vieira, S.D.; da Cunha Vieira Perini, F.; de Sousa, L.C.B.; Buffolo, E.; Chaccur, P.; Arrais, M.; Jatene, F.B. Autologous blood salvage in cardiac surgery: Clinical evaluation, efficacy and levels of residual heparin. Hematol. Transfus. Cell Ther. 2021, 43, 1-8. [CrossRef] [PubMed]

32. Smith, T.O.; Hing, C.B. Is a tourniquet beneficial in total knee replacement surgery? A meta-analysis and systematic review. Knee 2010, 17, 141-147. [CrossRef] [PubMed]

33. Spahn, D.R.; Shander, A.; Hofmann, A. The chiasm: Transfusion practice versus patient blood management. Best Pract. Res. Clin. Anaesthesiol. 2013, 27, 37-42. [CrossRef] [PubMed] 